\title{
Correction to: Microstructure, mechanical, in vitro corrosion and biocompatibility response study of as-cast and as-rolled Mg-5Zn-0.5Zr alloy
}

\author{
Anuradha Jana $^{1,2} \cdot$ Sourav Dutta $^{3} \cdot$ Mangal Roy $^{4} \cdot$ U. Aravind ${ }^{5} \cdot$ Mitun Das $^{1,2}\left(\mathbb{D}^{\circ} \cdot\right.$ Vamsi Krishna Balla $^{1,2}$
}

Published online: 8 March 2022

(c) The Author(s), under exclusive licence to The Materials Research Society 2022

\section{Correction to: MRS Advances (2021) 6:472-476 \\ https://doi.org/10.1557/s43580-021-00056-7}

This article was updated to correct affiliation 2.

The original article can be found online at https://doi.org/10.1557/ s43580-021-00056-7.

\section{Mitun Das}

mitun@cgcri.res.in

$\triangle$ Vamsi Krishna Balla vamsiballa@cgcri.res.in

1 CSIR-Central Glass and Ceramic Research Institute (CGCRI), Kolkata 700032, India

2 Academy of Scientific and Innovative Research (AcSIR), Ghaziabad 201002, India

3 Advanced Technology Development Centre, IIT Kharagpur, Kharagpur 721302, India

4 Department of Metallurgical and Materials Engineering, IIT Kharagpur, Kharagpur 721302, India

5 Department of Metallurgical and Materials Engineering, IIT Madras, Chennai 600 036, India 